\title{
Valeurs moyennes de fonctions multiplicatives sur les entiers friables translatés
}

\author{
par \\ Joseph BASQuin (Nancy)
}

\section{Introduction}

1.1. Contexte. Désignons par $P(n)$ le plus grand facteur premier d'un entier naturel positif $n$, avec la convention $P(1)=1$, et par

$$
S(x, y):=\{n \leq x: P(n) \leq y\}
$$

l'ensemble des entiers $y$-friables n'excédant pas $x$. Pour $x, y \geq 2$, nous posons, conformément à l'usage,

$$
\Psi(x, y):=|S(x, y)| \quad \text { et } \quad u:=(\log x) / \log y .
$$

Étant donnée une fonction arithmétique $f$, nous introduisons les quantités

$$
\Psi_{f}(x, y):=\sum_{n \in S(x, y)} f(n), \quad \Theta_{f}(x, y):=\sum_{\substack{n \in S(x, y) \\ n>1}} f(n-1) .
$$

La comparaison de $\Theta_{f}$ et $\Psi_{f}$ est un exemple de question concernant l'effet d'une perturbation additive, ici $n \mapsto n-1$ (et plus généralement $n \mapsto$ $n+a$, avec $a$ un entier non nul), sur une suite définie par des contraintes multiplicatives. Un autre exemple est le problème classique des diviseurs de Titchmarsh $\left({ }^{1}\right)$, qui consiste à évaluer asymptotiquement la quantité $\sum_{p \leq x} \tau(p-1)$, où $p$ désigne systématiquement un nombre premier et $\tau$ est la fonction nombre de diviseurs.

Fouvry et Tenenbaum [3] ont considéré les quantités (1) dans le cas où $f=\tau$. Ils ont mis en lumière une divergence radicale entre les quantités

2010 Mathematics Subject Classification: 11N25, 11N37.

Key words and phrases: friable integers, shifted friable integers, Euler function, multiplicative functions, sums of multiplicative functions, distribution of friable integers in arithmetic progressions.

$\left({ }^{1}\right)$ Voir notamment les travaux de Fouvry 2] pour des estimations précises à ce sujet. 
$\Theta_{\tau}(x, y)$ et $\Psi_{\tau}(x, y)$. Alors qu'il est établi dans [3] que

$$
\Theta_{\tau}(x, y)=\Psi(x, y) \log x\left\{1+O\left(\frac{\log (u+1)}{\log y}\right)\right\} \quad\left(x^{c_{1} \log _{3} x / \log _{2} x} \leq y \leq x\right),
$$

il découle des résultats de Smida [9] ou de Tenenbaum et Wu [11, Corollaire 2.3] que l'estimation

$$
\Psi_{\tau}(x, y)=\Psi(x, y)(\log x) \frac{\varrho_{2}(u)}{u \varrho(u)}\left\{1+O\left(\frac{\log (u+1)}{\log y}\right)\right\}
$$

est valable dans le domaine

$$
x \geq 3, \quad \exp \left\{\left(\log _{2} x\right)^{5 / 3+\varepsilon}\right\} \leq y \leq x,
$$

où, pour $\kappa>0$, nous notons $\varrho_{\kappa}$ l'unique fonction continue sur $] 0, \infty[$ et dérivable sur $[1, \infty[$ satisfaisant à

$$
\begin{cases}\varrho_{\kappa}(u)=u^{\kappa-1} / \Gamma(\kappa) & (0<u \leq 1), \\ u \varrho_{\kappa}^{\prime}(u)+(1-\kappa) \varrho_{\kappa}(u)+\kappa \varrho_{\kappa}(u-1)=0 & (u>1)\end{cases}
$$

et $\varrho:=\varrho_{1}$. On déduit de la relation (3.10) de [8] que le facteur dépendant de $u$ dans le membre de droite de (3) vaut $\left({ }^{2}\right)$

$$
\frac{\varrho_{2}(u)}{u \varrho(u)}=2^{u+O(u / \log (u+1))} \quad(u \rightarrow \infty) .
$$

Dans le même esprit, Loiperdinger et Shparlinski [7] ont récemment étudié la fonction sommatoire de la fonction indicatrice d'Euler $n \mapsto \varphi(n)$ sur les entiers friables translatés, soit

$$
\Theta_{\varphi}(x, y)=\sum_{\substack{n \in S(x, y) \\ n>1}} \varphi(n-1) .
$$

Ils obtiennent le résultat suivant.

THÉORÈme A. Il existe une constante $c_{1}>0$ telle que la relation

$$
\Theta_{\varphi}(x, y)=\frac{3}{\pi^{2}} x \Psi(x, y)\left\{1+O\left(\frac{\left(\log _{2} x\right) \log _{2} y}{\log y}\right)\right\}
$$

ait lieu uniformément dans le domaine

$$
x \geq x_{0}, \quad \exp \left\{c_{1} \sqrt{(\log x) \log _{3} x}\right\} \leq y \leq x .
$$

Nous nous proposons ici de préciser et généraliser ce résultat sous forme d'une estimation générale de la valeur moyenne de certaines fonctions multiplicatives sur les entiers friables translatés. Nos résultats englobent le cas de la fonction indicatrice d'Euler.

$\left(^{2}\right)$ Voir [6] pour un développement asymptotique des quantités $\varrho_{\kappa}$, et plus généralement, des solutions de certaines équations différentielles aux différences analogues à (4). 
1.2. Notations et définitions additionnelles. Les fonctions de Möbius, somme des diviseurs et identité sont notées respectivement $\mu, \sigma$ et $j$.

Nous définissons également

$$
\begin{aligned}
\Psi_{d}(x, y) & :=|\{n \in S(x, y):(n, d)=1\}|, \\
\Psi(x, y ; a, d) & :=|\{n \in S(x, y): n \equiv a \bmod d\}|
\end{aligned}
$$

et notons

$$
E(x, y ; a, d):=\Psi(x, y ; a, d)-\Psi_{d}(x, y) / \varphi(d) .
$$

Nous disons qu'un produit infini $\prod_{n \geq 1} a_{n}$ est convergent s'il existe un entier $N \geq 1$ tel que $P_{N}:=\lim _{k \rightarrow \infty} \prod_{N<n \leq k} a_{n}$ soit fini et non nul; nous posons alors $\prod_{n \geq 1} a_{n}:=\left(\prod_{n \leq N} a_{n}\right) P_{N}$.

Étant donnée une fonction arithmétique $f$, nous écrivons formellement

$$
\begin{aligned}
E_{f}(p) & :=\left(1-\frac{1}{p}\right) \sum_{\nu \geq 0} \frac{f\left(p^{\nu}\right)}{p^{\nu}} \quad(p \geq 2), \\
C(f) & :=\prod_{p} E_{f}(p) .
\end{aligned}
$$

1.3. Énoncé des résultats. Afin d'évaluer la quantité $\Theta_{f}(x, y)$, nous introduisons la fonction $\lambda:=f * \mu$. Remarquons que

$$
\Theta_{f}(x, y)=\sum_{d \leq x} \lambda(d) \Psi(x, y ; 1, d) \quad(x, y \geq 2) .
$$

Compte tenu des résultats de la littérature sur la répartition des entiers friables dans les progressions arithmétiques, on s'attend à une relation asymptotique du type

$$
\Theta_{f}(x, y) \approx \sum_{d \leq x} \frac{\lambda(d)}{\varphi(d)} \Psi_{d}(x, y) \approx \Psi(x, y) \sum_{d \leq x} \frac{\lambda(d)}{d} .
$$

Dans le cas où la fonction multiplicative $f$ est suffisamment proche dans un sens à préciser ultérieurement - de la fonction 1, il est raisonnable de penser que la fonction $\lambda$ est petite en valeur absolue, de sorte que, avec la notation (5),

$$
\sum_{d \geq 1} \frac{\lambda(d)}{d}=C(f)
$$

est bien définie. Nous pouvons alors conjecturer que, sous des hypothèses adéquates concernant $f$, nous avons

$$
\Theta_{f}(x, y) \approx C(f) \Psi(x, y) \approx \Psi_{f}(x, y) .
$$

Guidé par ce raisonnement heuristique, nous obtenons le résultat suivant. 
ThÉORÈme 1. Soient $\beta>0, B>0$, et $f$ une fonction multiplicative vérifiant

$$
|\lambda(d)| \leq \frac{B}{d^{\beta}} .
$$

Alors le produit $C(f)$ converge et, pour tous $x, y$ satisfaisant $\left(\mathrm{H}_{\varepsilon}\right)$, nous avons

$$
\Theta_{f}(x, y)=\Psi(x, y)\left\{C(f)+O\left(\frac{\log (u+1)}{\log y}\right)\right\},
$$

où la constante implicite dépend au plus de $\beta$ et $B$.

Corollaire 2. Sous les mêmes hypothèses, posant $F(n):=n f(n)$ $(n \geq 1)$, nous avons, pour tous $x, y$ satisfaisant $\left(\mathrm{H}_{\varepsilon}\right)$,

$$
\Theta_{F}(x, y)=x \Psi(x, y)\left\{\frac{1}{2} C(f)+O\left(\frac{\log (u+1)}{\log y}\right)\right\} .
$$

Le corollaire suivant étend et précise le Théorème A. Nous posons

$$
\begin{gathered}
\varphi_{1}(n):=(-1)^{n-1} \varphi(n) \quad(n \geq 1), \\
f_{1}\left(2^{\nu}\right):=-\nu+1 \quad(\nu \geq 1), \quad f_{1}\left(p^{\nu}\right):=1 \quad(p \neq 2, \nu \geq 1) .
\end{gathered}
$$

Les fonctions $\varphi_{1}$ et $f_{1}$ fournissent des exemples d'application du Corollaire 2 au cas de fonctions de signe non constant ou de valeur moyenne nulle.

Corollaire 3. Pour tous $x$, y satisfaisant $\left(\mathrm{H}_{\varepsilon}\right)$, nous avons

$$
\begin{aligned}
\Theta_{\varphi}(x, y) & =\frac{3}{\pi^{2}} x \Psi(x, y)\left\{1+O\left(\frac{\log (u+1)}{\log y}\right)\right\}, \\
\Theta_{\sigma}(x, y) & =\frac{\pi^{2}}{12} x \Psi(x, y)\left\{1+O\left(\frac{\log (u+1)}{\log y}\right)\right\}, \\
\Theta_{\varphi_{1}}(x, y) & =\frac{1}{\pi^{2}} x \Psi(x, y)\left\{1+O\left(\frac{\log (u+1)}{\log y}\right)\right\}, \\
\Theta_{f_{1}}(x, y) & \ll \frac{\Psi(x, y) \log (u+1)}{\log y} .
\end{aligned}
$$

2. Résultats auxiliaires. Nous commençons par une estimation concernant certaines intégrales.

Lemme 4. Nous avons, uniformément pour $0 \leq \gamma \leq 1, \beta \in \mathbb{R}$ et $y \geq$ $w>1$,

$$
\int_{w}^{y} \frac{d t}{t^{\beta} \exp \left\{(\log t)^{\gamma}\right\}} \ll \int_{w}^{y} \frac{d t}{t \exp \left\{\left(\frac{1}{4} \log t\right)^{\gamma}\right\}}+\frac{y^{1-\beta}-1}{(1-\beta) \exp \left\{\left(\frac{1}{4} \log y\right)^{\gamma}\right\}} .
$$

Démonstration. L'estimation annoncée résulte du Lemme 3.2 de [11. Vérifions que les hypothèses en sont satisfaites. Soit $R(t):=\exp \left\{(\log t)^{\gamma}\right\}$ 
avec $0 \leq \gamma \leq 1$. Nous avons $\varphi(v):=\log R\left(e^{v}\right)=v^{\gamma}(v>0)$. La fonction $\varphi^{\prime}$ est décroissante sur ]0, $\infty$ [ et l'on a, pour tout $0 \leq \gamma \leq 1$,

$$
\frac{1}{2} \varphi^{\prime}\left(\frac{1}{2} v\right) \leq \frac{3}{4} \int_{1 / 4}^{1} \varphi^{\prime}(t v) d t \quad(v>0) .
$$

En effet, cette dernière condition équivaut à

$$
\frac{\gamma}{2^{\gamma}} \leq \frac{3}{4}\left(1-\frac{1}{4^{\gamma}}\right) \quad(0 \leq \gamma \leq 1)
$$

qui est bien vérifiée car

$$
4 \gamma / 3 \leq 2 \gamma \log 2 \leq 2 \operatorname{sh}(\gamma \log 2)=2^{\gamma}-2^{-\gamma} \quad(0 \leq \gamma \leq 1) .
$$

Le résultat découle alors du lemme précité appliqué avec $b:=1 / 4$.

Soit $\alpha=\alpha(x, y)$ défini comme l'unique solution positive de l'équation

$$
\sum_{p \leq y} \frac{\log p}{p^{\alpha}-1}=\log x
$$

Rappelons tout d'abord l'estimation classique (voir [10, formule (III.5.74)])

$$
1-\alpha(x, y) \ll \frac{\log (u+1)}{\log y} \quad\left(x \geq x_{0}(\varepsilon),(\log x)^{1+\varepsilon} \leq y \leq x\right) .
$$

Le résultat suivant, relatif au comportement local de $\Psi(x, y)$, est établi dans [1, Théorème 2.4].

Lemme 5. Il existe une constante $c_{2}>0$ telle que pour $x \geq 2, x \geq y \geq$ $\log x$ et $1 \leq z \leq T:=\exp \left\{c_{2} \sqrt{\log (u+1) \log x}\right\}$, nous ayons

$$
\Psi\left(\frac{x}{z}, y\right)=\frac{\Psi(x, y)}{z^{\alpha}}\left(1+O\left(\frac{\log (u+1)}{\log y}+\frac{\log z}{\log x}\right)\right) .
$$

Par ailleurs, pour $x \geq y \geq 2$ et $1 \leq z \leq x$, nous avons

$$
\Psi\left(\frac{x}{z}, y\right) \ll \frac{\Psi(x, y)}{z^{\alpha}} .
$$

Pour $A>0, C>0, \varepsilon>0$ et $\eta \in] 0,1 / 2[$, nous posons

$$
L_{\varepsilon}(x):=\exp \left\{(\log x)^{3 / 5-\varepsilon}\right\} \quad(x \geq 1)
$$

et nous désignons par $\mathcal{M}_{1}(A, C, \eta, \varepsilon)$ la classe des fonctions multiplicatives réelles positives ou nulles $f$ satisfaisant aux conditions

$$
\begin{aligned}
\left|\sum_{p \leq z} f(p) \log p-z\right| & \leq \frac{C z}{L_{\varepsilon}(z)} \quad(z \geq 2), \\
\sum_{p} \sum_{\nu \geq 2} \frac{\left|f\left(p^{\nu}\right)\right|}{p^{(1-\eta) \nu}} & \leq A .
\end{aligned}
$$


De même, nous notons $\mathcal{M}^{ \pm}(A, C, \eta, \varepsilon)$ la classe des fonctions multiplicatives réelles $f$ vérifiant la condition 10 et

$$
\sum_{\substack{p \leq z \\ f(p)<0}}|f(p)| \log p+\left|\sum_{p \leq z} f(p) \log p-z\right| \leq \frac{C z}{L_{\varepsilon}(z)} \quad(z \geq 2) .
$$

Le résultat suivant est une extension à certaines fonctions multiplicatives réelles du cas particulier $\kappa=1$ du Corollaire 2.3 de [11].

Lemme 6. Soient $A>0, C>0, \varepsilon>0$ et $\eta \in] 0,1 / 2[$. Pour tout $f \in \mathcal{M}^{ \pm}(A, C, \eta, \varepsilon)$, le produit $C(f)$ converge et nous avons, uniformément pour $(x, y) \in\left(\mathrm{H}_{\varepsilon}\right)$ et $f \in \mathcal{M}^{ \pm}(A, C, \eta, \varepsilon / 2)$,

$$
\Psi_{f}(x, y)=\Psi(x, y)\left\{C(f)+O\left(\frac{\log (u+1)}{\log y}\right)\right\} .
$$

Démonstration. Le cas où la fonction $f$ est positive ou nulle est établi au Corollaire 2.3 de [11].

Soient $g$ et $h$ les fonctions multiplicatives définies $\operatorname{par}\left({ }^{3}\right)$

$$
g\left(p^{\nu}\right)=\left\{f(p)^{+}\right\}^{\nu} / \nu ! \quad(p \geq 2, \nu \geq 1), \quad f=g * h .
$$

Montrons tout d'abord que pour $(x, y) \in\left(\mathrm{H}_{\varepsilon}\right)$, nous avons

$$
\sum_{\substack{p \leq y \\ \nu \geq 1}} \frac{\left|h\left(p^{\nu}\right)\right|\left(\log p^{\nu}\right)^{j}}{p^{\nu \alpha}} \ll 1 \quad(j \in\{0,1,2\}) .
$$

Il est suffisant d'établir le cas $j=2$. Observons que $h(p)=-f(p)^{-}$. Par sommation d'Abel et compte tenu de la condition (11), il suit, pour $(x, y) \in\left(\mathrm{H}_{\varepsilon}\right)$,

$$
\begin{aligned}
U_{1} & :=\sum_{p \leq y} \frac{|h(p)| \log p}{p^{\alpha}}=\sum_{\substack{p \leq y \\
f(p)<0}} \frac{|f(p)| \log p}{p^{\alpha}} \ll \int_{1}^{y} \frac{d z}{z^{\alpha} L_{\varepsilon / 2}(z)} \\
& \ll 1+\frac{y^{1-\alpha}-1}{(1-\alpha) L_{\varepsilon / 2}\left(y^{1 / 4}\right)},
\end{aligned}
$$

en vertu du Lemme 4. L'inégalité $\left(e^{r}-1\right) / r \leq e^{r}(r>0)$ permet alors d'établir

$$
U_{1} \ll 1+y^{1-\alpha} \frac{\log y}{L_{\varepsilon / 2}\left(y^{1 / 4}\right)} \ll 1+\frac{\log x}{L_{\varepsilon / 2}\left(y^{1 / 4}\right)} \ll 1 \quad\left((x, y) \in\left(\mathrm{H}_{\varepsilon}\right)\right),
$$

compte tenu de (7). D'autre part, l'expression explicite de $h$, donnée par

$$
h\left(p^{\nu}\right)=\sum_{j+k=\nu}(-1)^{j} f\left(p^{k}\right)\left\{f(p)^{+}\right\}^{j} / j ! \quad(p \geq 2, \nu \geq 1),
$$

$\left({ }^{3}\right)$ Pour $a \in \mathbb{R}$, nous notons $a^{+}:=\max \{a, 0\}$ et $a^{-}:=\min \{a, 0\}$. 
nous permet d'effectuer la majoration

$$
\sum_{\nu \geq 2} \frac{\left|h\left(p^{\nu}\right)\right|\left(\log p^{\nu}\right)^{2}}{p^{\nu \alpha}} \leq \sum_{k \geq 0} \frac{\left|f\left(p^{k}\right)\right|(\log p)^{2}}{p^{k \alpha}} \sum_{j \geq \max \{0,2-k\}} \frac{\left\{f(p)^{+}\right\}^{j}(j+k)^{2}}{j ! p^{j \alpha}} .
$$

Désignons par $S_{k}$ la somme intérieure. La relation

$$
f(p) \ll \frac{p}{L_{\varepsilon / 2}(p) \log p},
$$

découlant directement de la condition (9), nous assure que l'on a, pour $p \leq y$,

$$
\begin{aligned}
\frac{f(p)}{p^{\alpha}} & \ll \exp \left\{(1-\alpha) \log p-(\log p)^{3 / 5-\varepsilon / 2}\right\} \\
& \ll \exp \left\{(\log p)^{3 / 5-\varepsilon / 2}\left((1-\alpha)(\log y)^{2 / 5+\varepsilon / 2}-1\right)\right\} .
\end{aligned}
$$

Nous avons donc, grâce à l'estimation (7),

$$
\frac{f(p)}{p^{\alpha}} \ll 1 \quad\left((x, y) \in\left(\mathrm{H}_{\varepsilon}\right), p \leq y\right) .
$$

Posons $\xi:=f(p)^{+} / p^{\alpha}$. Nous pouvons à présent établir les majorations

$$
\begin{aligned}
& S_{0}=\xi\left(e^{\xi}-1+\xi e^{\xi}\right) \ll \xi^{2} \leq f(p)^{2} / p^{2 \alpha}, \\
& S_{1}=e^{\xi}-1+3 \xi e^{\xi}+\xi^{2} e^{\xi} \ll \xi \leq|f(p)| / p^{\alpha}, \\
& S_{k}=e^{\xi}\left(k^{2}+2 \xi k+\xi+\xi^{2}\right) \ll k^{2} \quad(k \geq 2) .
\end{aligned}
$$

Nous en déduisons

$$
\sum_{\substack{p \leq y \\ \nu \geq 1}} \frac{\left|h\left(p^{\nu}\right)\right|\left(\log p^{\nu}\right)^{2}}{p^{\nu \alpha}} \ll 1+\sum_{p \leq y} \frac{f(p)^{2}(\log p)^{2}}{p^{2 \alpha}}+\sum_{\substack{p \leq y \\ k \geq 2}} \frac{\left|f\left(p^{k}\right)\right|\left(\log p^{k}\right)^{2}}{p^{k \alpha}} .
$$

Posons $N(t):=\sum_{p \leq t} f(p) \log p$. Les relations (11) et (13) impliquent alors que

$$
\begin{aligned}
& \sum_{p \leq y} \frac{f(p)^{2}(\log p)^{2}}{p^{2 \alpha}} \ll \sum_{p \leq y} \frac{f(p) \log p}{p^{2 \alpha-1} L_{\varepsilon / 2}(p)}=\int_{2^{-}}^{y} \frac{d N(t)}{t^{2 \alpha-1} L_{\varepsilon / 2}(t)} \\
& =\left[\frac{N(t)}{t^{2 \alpha-1} L_{\varepsilon / 2}(t)}\right]_{2^{-}}^{y}+\int_{2^{-}}^{y} \frac{1-2 \alpha-(3 / 5-\varepsilon / 2)(\log t)^{-2 / 5-\varepsilon / 2}}{t^{2 \alpha} L_{\varepsilon / 2}(t)} N(t) d t \\
& \ll \frac{y^{2-2 \alpha}}{L_{\varepsilon / 2}(y)}+\int_{2}^{y} \frac{t^{1-2 \alpha}}{L_{\varepsilon / 2}(t)} d t \ll 1+\frac{y^{2-2 \alpha}-1}{(1-\alpha) L_{\varepsilon / 2}\left(y^{1 / 4}\right)} \ll 1,
\end{aligned}
$$

pour $(x, y) \in\left(\mathrm{H}_{\varepsilon}\right)$, par les mêmes arguments que ceux qui nous ont conduit à la majoration de la quantité $U_{1}$.

Il existe une constante $c_{3}>0$ telle que, pour $x, y$ assez grands dans le domaine $\left(\mathrm{H}_{\varepsilon}\right)$, nous ayons $\alpha-(1-\eta) \geq c_{3}$. Par conséquent, la seconde 
somme du membre de droite de (14) est majorée par

$$
\sum_{\substack{p \leq y \\ k \geq 2}} \frac{\left|f\left(p^{k}\right)\right|}{p^{(1-\eta) k}} \frac{\left(\log p^{k}\right)^{2}}{p^{c_{3} k}} \leq c_{4} A
$$

où

$$
c_{4}:=\sup _{t \geq 1} \frac{(\log t)^{2}}{t^{c_{3}}} .
$$

Ceci achève la démonstration de la majoration (12).

Montrons à présent que pour $(x, y) \in\left(\mathrm{H}_{\varepsilon}\right)$, l'on a

$$
\sum_{P(m) \leq y} \frac{|h(m)|(\log m)^{j}}{m^{\alpha}} \ll 1 \quad(j \in\{0,1,2\}) .
$$

Remarquant que le cas $j=1$ découle naturellement des cas $j=0$ et 2 , il est suffisant de démontrer ceux-ci. Le cas $j=0$ de 12 implique directement le cas $j=0$ de (16) par application du Théorème II.1.3 de [10]. Pour établir le cas $j=2$ de (16), nous observons que

$$
(\log m)^{2}=\sum_{p^{\nu} \| m}\left(\log p^{\nu}\right)^{2}+\sum_{\substack{p^{\nu} q^{\mu} \| m \\ p \neq q}}\left(\log p^{\nu}\right)\left(\log q^{\mu}\right) \quad(m \geq 1) .
$$

Ceci nous permet d'écrire

$$
\begin{aligned}
\sum_{P(m) \leq y} \frac{|h(m)|(\log m)^{2}}{m^{\alpha}} \leq & \sum_{\substack{p \leq y \\
\nu \geq 1}} \frac{\left|h\left(p^{\nu}\right)\right|\left(\log p^{\nu}\right)^{2}}{p^{\nu \alpha}} \sum_{P(m) \leq y} \frac{|h(m)|}{m^{\alpha}} \\
& +\left(\sum_{\substack{p \leq y \\
\nu \geq 1}} \frac{\left|h\left(p^{\nu}\right)\right| \log p^{\nu}}{p^{\nu \alpha}}\right)^{2} \sum_{P(m) \leq y} \frac{|h(m)|}{m^{\alpha}} \ll 1,
\end{aligned}
$$

par application de 12 pour $j=1$ et 2 , ce qui fournit l'estimation 16 pour $j=2$.

L'intégralité des estimations précédentes reste valable, en remplaçant formellement dans les formules le paramètre $\alpha$ par 1 et en faisant tendre $y$ vers l'infini. Cela résulte immédiatement du fait que les intégrales du type $\int_{1}^{\infty} d z /\left\{z L_{\varepsilon / 2}(z)\right\}$ sont convergentes. Nous en déduisons

$$
\sum_{m \geq 1} \frac{|h(m)|(\log m)^{j}}{m} \ll 1 \quad(j \in\{0,1,2\}) .
$$


La méthode de Rankin permet de montrer que, pour tout $V \geq 2$,

$$
\begin{gathered}
\sum_{\substack{m>V \\
P(m) \leq y}} \frac{|h(m)|}{m^{\alpha}} \leq \sum_{P(m) \leq y} \frac{|h(m)|(\log m)^{2}}{m^{\alpha}(\log V)^{2}} \ll \frac{1}{(\log V)^{2}}, \\
\sum_{m>V} \frac{|h(m)|}{m} \leq \sum_{m \geq 1} \frac{|h(m)|(\log m)^{2}}{m(\log V)^{2}} \ll \frac{1}{(\log V)^{2}} .
\end{gathered}
$$

Par ailleurs, l'inégalité des accroissements finis et la relation (7) impliquent que, pour $(x, y) \in\left(\mathrm{H}_{\varepsilon}\right)$,

$$
\sum_{P(m) \leq y}|h(m)|\left\{\frac{1}{m^{\alpha}}-\frac{1}{m}\right\} \leq \sum_{P(m) \leq y} \frac{|h(m)|(1-\alpha) \log m}{m^{\alpha}} \ll \frac{\log (u+1)}{\log y} .
$$

Nous sommes à présent en mesure d'évaluer la somme annoncée. Nous avons

$$
\Psi_{f}(x, y)=\sum_{m \in S(x, y)} h(m) \Psi_{g}\left(\frac{x}{m}, y\right) .
$$

Si $f \in \mathcal{M}^{ \pm}(A, C, \eta, \varepsilon / 2)$, il est clair que $g \in \mathcal{M}_{1}(A, C, \eta, \varepsilon / 2)$ et nous pouvons ainsi utiliser le résultat déjà établi pour cette classe - comme rappelé en début de preuve - qui fournit, pour $x, y$ satisfaisant $\left(\mathrm{H}_{\varepsilon}\right)$ et $1 \leq m \leq x$,

$$
\Psi_{g}\left(\frac{x}{m}, y\right)=C(g) \Psi\left(\frac{x}{m}, y\right)\left\{1+O\left(\frac{\log (u+1)}{\log y}+\frac{1}{\log (2 x / m)}\right)\right\} .
$$

D'autre part, nous avons pour $x \geq 2, y \geq \log x$ et $m \leq T:=\exp \{\sqrt{\log x}\}$, grâce au Lemme 5 .

$$
\Psi\left(\frac{x}{m}, y\right)=\frac{\Psi(x, y)}{m^{\alpha}}\left\{1+O\left(\frac{\log (u+1)}{\log y}+\frac{\log m}{\log x}\right)\right\} .
$$

Posons, sous réserve de convergence,

$$
H(s):=\sum_{m \geq 1} \frac{h(m)}{m^{s}}, \quad H_{T}(s ; y):=\sum_{m \in S(T, y)} \frac{h(m)}{m^{s}} .
$$

La relation 21] nous permet d'écrire $\Psi_{f}(x, y)=\Sigma_{1}+\Sigma_{2}$ avec

$$
\Sigma_{1}:=\sum_{m \in S(T, y)} h(m) \Psi_{g}\left(\frac{x}{m}, y\right), \quad \Sigma_{2}:=\sum_{\substack{m \in S(x, y) \\ m>T}} h(m) \Psi_{g}\left(\frac{x}{m}, y\right) .
$$

Il s'ensuit, grâce 22 et 23 , que, pour $(x, y) \in\left(\mathrm{H}_{\varepsilon}\right)$,

$$
\Sigma_{1}=C(g) H_{T}(\alpha ; y) \Psi(x, y)\left(1+O\left(\frac{\log (u+1)}{\log y}\right)\right)+O\left(\frac{\Psi(x, y)}{\log x}\right),
$$


d'après (16) pour $j=1$. Les majorations issues de (8) et $(18)$ fournissent l'évaluation pleinement suffisante

$$
\Sigma_{2} \ll \Psi(x, y) \sum_{\substack{m>T \\ P(m) \leq y}} \frac{|h(m)|}{m^{\alpha}} \ll \frac{\Psi(x, y)}{\log y} .
$$

Soit $T_{y}:=\min \{T, y\}$. Nous avons alors

$$
\begin{aligned}
\left|H_{T}(\alpha ; y)-H(1)\right| & =\left|\sum_{\substack{m \leq T \\
P(m) \leq y}} h(m)\left\{\frac{1}{m^{\alpha}}-\frac{1}{m}\right\}-\sum_{m>T \text { ou } P(m)>y} \frac{h(m)}{m}\right| \\
& \leq \sum_{P(m) \leq y}|h(m)|\left\{\frac{1}{m^{\alpha}}-\frac{1}{m}\right\}+\sum_{m>T_{y}} \frac{|h(m)|}{m} \\
& \ll \frac{\log (u+1)}{\log y}+\frac{1}{\log x}+\frac{1}{(\log y)^{2}} \ll \frac{\log (u+1)}{\log y},
\end{aligned}
$$

d'après $(20)$ et 190 .

Montrons à présent que le produit $C(f)$ converge. Remarquons tout d'abord que l'on peut écrire $E_{f}(p)=1+u_{p}+v_{p}$, où

$$
u_{p}:=\frac{f(p)-1}{p}, \quad v_{p}:=\sum_{\nu \geq 2} \frac{f\left(p^{\nu}\right)-f\left(p^{\nu-1}\right)}{p^{\nu}} \quad(p \geq 2) .
$$

Le Lemme III.4.3 de [10] nous assure que, sous les hypothèses $\sum_{p} u_{p}^{2}<\infty$, $\sum_{p}\left|v_{p}\right|<\infty$, une condition nécessaire et suffisante pour que le produit $C(f)$ converge est la convergence de la série $\sum_{p} u_{p}$.

D'une part, l'inégalité $(a+b)^{2} \leq 2\left(a^{2}+b^{2}\right)(a, b \in \mathbb{R})$ et le fait que l'on ait $\sum_{p} f(p)^{2} / p^{2}<\infty$ (la démonstration est en tout point similaire à celle de (15)) impliquent que la série $\sum_{p} u_{p}^{2}$ est convergente. Il en est de même de la série $\sum_{p} u_{p}$, ce qu'on établit par sommation d'Abel grâce à la condition (11). avons

D'autre part, la série $\sum_{p} v_{p}$ est absolument convergente. En effet, nous

$$
\left|v_{p}\right| \leq|f(p)| / p^{2}+2 \sum_{\nu \geq 2}\left|f\left(p^{\nu}\right)\right| / p^{\nu} \quad(p \geq 2) .
$$

La relation $\sum_{p}|f(p)| / p^{2}<\infty$ découle de la majoration (13), et la convergence absolue de la série $\sum_{p} v_{p}$ résulte ainsi de 10. Le Lemme précité assure donc bien la convergence du produit $C(f)$.

Enfin, nous avons bien $C(g) H(1)=C(f)$, puisque

$$
\sum_{\nu \geq 0} \frac{g\left(p^{\nu}\right)}{p^{\nu}} \sum_{\nu \geq 0} \frac{h\left(p^{\nu}\right)}{p^{\nu}}=\sum_{\nu \geq 0} \frac{f\left(p^{\nu}\right)}{p^{\nu}} \quad(p \geq 2) .
$$


Finalement, nous avons, pour $x, y$ satisfaisant $\left(\mathrm{H}_{\varepsilon}\right)$,

$$
\Psi_{f}(x, y)=\Psi(x, y)\left\{C(f)+O\left(\frac{\log (u+1)}{\log y}\right)\right\} .
$$

Le résultat suivant est nécessaire pour établir le Corollaire 2, et plus généralement pour étudier la somme friable d'une fonction proche de l'identité.

Lemme 7. Soit $\varepsilon>0$. Pour $(\log x)^{1+\varepsilon} \leq y \leq x$ et $x \geq x_{0}(\varepsilon)$, nous avons uniformément

$$
\begin{aligned}
\int_{1}^{x} \Psi(t, y) d t & =\frac{1}{2} x \Psi(x, y)\left\{1+O\left(\frac{\log (u+1)}{\log y}\right)\right\}, \\
\sum_{n \in S(x, y)} n & =\frac{1}{2} x \Psi(x, y)\left\{1+O\left(\frac{\log (u+1)}{\log y}\right)\right\} .
\end{aligned}
$$

Démonstration. Une intégration par parties donne, pour $x, y \geq 2$,

$$
\sum_{n \in S(x, y)} n=x \Psi(x, y)-\int_{1}^{x} \Psi(t, y) d t=x \Psi(x, y)-x \int_{1}^{x} \Psi\left(\frac{x}{z}, y\right) \frac{d z}{z^{2}} .
$$

Le Lemme 5 fournit, pour $x \geq y \geq \log x$ et $z \leq T=\exp \left\{c_{2} \sqrt{\log (u+1) \log x}\right\}$,

$$
\begin{aligned}
\int_{1}^{T} \Psi\left(\frac{x}{z}, y\right) \frac{d z}{z^{2}} & =\Psi(x, y)\left\{1+O\left(\frac{\log (u+1)}{\log y}\right)\right\} \int_{1}^{T} \frac{d z}{z^{2+\alpha}}+O\left(\frac{\Psi(x, y)}{\log x}\right) \\
& =\frac{\Psi(x, y)}{1+\alpha}\left\{1+O\left(\frac{\log (u+1)}{\log y}+\frac{1}{T}\right)\right\} .
\end{aligned}
$$

Par ailleurs, nous avons trivialement

$$
\int_{T}^{x} \Psi\left(\frac{x}{z}, y\right) \frac{d z}{z^{2}} \ll \frac{\Psi(x, y)}{T} \ll \frac{\Psi(x, y) \log (u+1)}{\log y} .
$$

Enfin, l'évaluation

$$
\frac{1}{1+\alpha}=\frac{1}{2}+O\left(\frac{\log (u+1)}{\log y}\right),
$$

valable pour $(\log x)^{1+\varepsilon} \leq y \leq x$ et $x \geq x_{0}(\varepsilon)$, découle de (7). Cela implique bien le résultat annoncé.

Le résultat suivant est une reformulation du Théorème 2 de [4].

Lemme 8. Il existe des constantes $c_{5}>0, c_{6}>0$ telles que, pour $x, y \geq 2$,

$$
\sum_{d \leq z}|E(x, y ; 1, d)| \ll \frac{\Psi(x, y)}{\log x},
$$

où $z:=\min \left\{\sqrt{x} /(\log x)^{c_{6}}, y^{c_{5} \log _{2} y / \log _{3} y}\right\}$. 
Le lemme suivant fournit une estimation du nombre des entiers friables dans les progressions arithmétiques de grands modules. Nous suivons la méthode employée pour établir la Proposition 1 de [4] et donnons une version légèrement modifiée du Théorème 4 de [4].

Lemme 9. Nous avons uniformément, pour $x, y$ satisfaisant $\left(\mathrm{H}_{\varepsilon}\right), 1 \leq$ $q \leq x$ et $(a, q)=1$,

$$
\Psi(x, y ; a, q) \ll \frac{\Psi(x, y)}{q \varrho\left(u_{q}\right)},
$$

où nous avons posé $u_{q}:=\min \{(\log q y) / \log y, u\}$.

Remarque. Notons que pour $q \geq x / y$, la méthode employée ici ne peut fournir davantage que la majoration triviale $\Psi(x, y ; a, q) \ll x / q$.

Démonstration du Lemme 9. Soit $x_{0} \geq 2$ et $x_{1} \geq 2 x_{0}$. Posons, pour $x \geq x_{0}, y \geq 2$ et $1 \leq q \leq x$,

$$
\Delta(x):=\max _{(a, q)=1} \frac{\Psi(x, y ; a, q)}{\Psi(x, y)}, \quad \Delta^{*}(x):=\max _{x_{0} \leq z \leq x} \Delta(z) .
$$

En évaluant les sommes

$$
\sum_{n \in S(x, y)} \log n \quad \text { et } \sum_{\substack{n \in S(x, y) \\ n \equiv a \bmod q}} \log n
$$

de deux manières différentes, nous obtenons les identités classiques (voir [4] et [5])

$$
\begin{gathered}
\Psi(x, y) \log x=\int_{1}^{x} \frac{\Psi(t, y)}{t} d t+\sum_{\substack{p \leq y \\
p^{m} \leq x}} \Psi\left(x / p^{m}, y\right) \log p, \\
\Psi(x, y ; a, q) \log x=\int_{1}^{x} \frac{\Psi(t, y ; a, q)}{t} d t+\sum_{\substack{p \leq y \\
p^{m} \leq x}}\left(\sum_{\substack{k \in S\left(x / p^{m}, y\right) \\
k p^{m} \equiv a \bmod q}} 1\right) \log p .
\end{gathered}
$$

Si $p \nmid q$, nous avons, notant $p^{-m}$ l'inverse de $p^{m}$ modulo $q$,

$$
\sum_{\substack{k \in S\left(x / p^{m}, y\right) \\ k p^{m} \equiv a \bmod q}} 1=\Psi\left(x / p^{m}, y ; a p^{-m}, q\right)
$$

et, dans le cas contraire, cette somme est nulle car les relations $p \mid q$ et $k p^{m} \equiv a \bmod q$ impliquent $(a, q)>1$, ce qui contredit l'hypothèse faite sur $a$. Il vient alors, pour $x_{0}, q, y \leq x$, grâce à l'identité (26),

$$
\Delta(x) \Psi(x, y) \log x \leq \int_{x_{0}}^{x} \Delta(t) \frac{\Psi(t, y)}{t} d t+\sum_{\substack{p \leq y \\ p^{m} \leq x / x_{0}}} \Delta\left(x / p^{m}\right) \Psi\left(x / p^{m}, y\right) \log p+H
$$


où nous avons posé

$$
\begin{aligned}
H & :=\int_{1}^{x_{0}} \max _{(a, q)=1} \Psi(t, y ; a, q) \frac{d t}{t}+\sum_{\substack{p \leq y \\
x / x_{0}<p^{m} \leq x}} \max _{(a, q)=1} \Psi\left(x / p^{m}, y ; a, q\right) \log p \\
& \leq\left(\max _{(a, q)=1} \Psi\left(x_{0}, y ; a, q\right)\right)\left(\int_{1}^{x_{0}} \frac{1}{t} d t+\sum_{\substack{p \leq y \\
x / x_{0}<p^{m} \leq x}} \log p\right) .
\end{aligned}
$$

Remarquons que la relation

$$
\sum_{m \geq 1, x / x_{0}<p^{m} \leq x} 1 \leq \frac{\log x}{\log p}-\frac{\log \left(x / x_{0}\right)}{\log p}+O(1)=\frac{\log x_{0}}{\log p}+O(1)
$$

implique la majoration

$$
\sum_{\substack{p \leq y \\ x / x_{0}<p^{m} \leq x}} \log p \leq\left(\log x_{0}\right) \pi(y)+O(y) \ll \pi(y)\left(\log x_{0}+\log y\right) .
$$

Nous en déduisons ainsi que la quantité

$$
H \ll\left(\max _{(a, q)=1} \Psi\left(x_{0}, y ; a, q\right)\right)(\pi(y)+1) \log \left(x_{0} y\right)
$$

est bornée indépendamment de $x$. Nous avons, pour $x \geq 2 x_{0}$,

$$
\begin{aligned}
& \Delta(x) \Psi(x, y) \log x \\
& \leq \Delta^{*}(x / 2)\left\{\int_{x_{0}}^{x} \frac{\Psi(t, y)}{t} d t+\sum_{\substack{p \leq y \\
p^{m} \leq x / x_{0}}} \Psi\left(x / p^{m}, y\right) \log p\right\} \\
& \quad+\left\{\Delta^{*}(x)-\Delta^{*}(x / 2)\right\} \int_{x / 2}^{x} \frac{\Psi(t, y)}{t} d t+H \\
& \leq \Delta^{*}(x / 2) \Psi(x, y) \log x+\left\{\Delta^{*}(x)-\Delta^{*}(x / 2)\right\} \Psi(x, y) \log 2+H,
\end{aligned}
$$

où la dernière inégalité découle de l'identité (25). Pour $x \geq 2 x_{0} \geq 4$, nous obtenons

$$
\begin{aligned}
\Delta(x) & \leq \Delta^{*}(x / 2)+\left(\Delta^{*}(x)-\Delta^{*}(x / 2)\right) \frac{\log 2}{\log x}+\frac{H}{\Psi(x, y) \log x} \\
& \leq \frac{1}{2} \Delta^{*}(x / 2)+\frac{1}{2} \Delta^{*}(x)+\frac{H}{\Psi(x, y) \log x}
\end{aligned}
$$

car $2 \log 2 \leq \log x$.

Supposons à présent que $x \geq 4 x_{0}$. Pour tout $x / 2 \leq z \leq x$, nous pouvons donner la majoration 


$$
\begin{aligned}
\Delta(z) & \leq \frac{1}{2} \Delta^{*}(z / 2)+\frac{1}{2} \Delta^{*}(z)+\frac{H}{\Psi(z, y) \log z} \\
& \leq \frac{1}{2} \Delta^{*}(x / 2)+\frac{1}{2} \Delta^{*}(x)+\frac{H}{\Psi(x / 2, y) \log (x / 2)}
\end{aligned}
$$

D'autre part, pour tout $x_{0} \leq z \leq x / 2$, nous avons trivialement, par croissance de la fonction $\Delta^{*}$, la majoration $\Delta(z) \leq \Delta^{*}(x / 2) \leq \frac{1}{2} \Delta^{*}(x / 2)+$ $\frac{1}{2} \Delta^{*}(x)$.

Nous obtenons finalement, pour $x \geq 4 x_{0}$,

$$
\Delta^{*}(x) \leq \Delta^{*}(x / 2)+\frac{2 H}{\Psi(x / 2, y) \log (x / 2)} .
$$

En utilisant cette relation de façon récurrente, nous obtenons, pour $x \geq 4 x_{0}$,

$$
\Delta(x) \leq \Delta^{*}\left(x_{1}\right)+2 H \sum_{j \geq 0, x_{1} 2^{j} \leq x} \frac{1}{\Psi\left(x_{1} 2^{j}, y\right) \log \left(x_{1} 2^{j}\right)} .
$$

Le Lemme 5 permet de majorer la dernière somme par la quantité

$$
\frac{1}{\Psi\left(x_{1}, y\right) \log x_{1}} \sum_{j \geq 0} \frac{1}{2^{j \alpha(x, y)}} .
$$

L'estimation (7) nous assure ainsi que l'on a, pour $x \geq 4 x_{0}$ et $(x, y) \in\left(\mathrm{H}_{\varepsilon}\right)$,

$$
\Delta(x) \leq \Delta^{*}\left(x_{1}\right)+O\left(\frac{H}{\Psi\left(x_{1}, y\right) \log x_{1}}\right) .
$$

La majoration $\Psi(z, y ; a, q) \ll z / q$, valable pour $z \geq q$, appliquée dans l'intervalle $x_{0} \leq z \leq x_{1}$ nous permet d'obtenir, pour $x \geq 4 x_{0} \geq 4 q$,

$$
\Delta(x) \ll \frac{1}{q}\left\{\max _{x_{0} \leq z \leq x_{1}} \frac{z}{\Psi(z, y)}+\frac{x_{0} y \log \left(x_{0} y\right)}{\Psi\left(x_{1}, y\right) \log x_{1}}\right\} .
$$

Choisissons $x_{0}=q$ et $x_{1}=q y$. Il vient alors pour $x \geq q \max \{4, y\}$,

$$
\Delta(x) \ll \frac{1}{q} \max _{q \leq z \leq q y} \frac{z}{\Psi(z, y)} \ll \frac{1}{q \varrho\left(u_{q}\right)},
$$

compte tenu de l'estimation classique $\Psi(z, y) \asymp x \varrho(u)$ valide pour $(z, y)$ $\in\left(\mathrm{H}_{\varepsilon}\right)$.

Le cas $x \leq q \max \{4, y\}$ se traite par la majoration triviale $\Psi(x, y ; a, q)$ $\ll x / q$.

Lemme 10. Nous avons uniformément, pour $\beta>0$ et $z \geq 1$,

$$
\sum_{n>z} \frac{1}{n^{\beta} \varphi(n)} \ll \frac{1}{\beta z^{\beta}} .
$$


Démonstration. L'identité $\sum_{d \mid n} \mu(d)^{2} / \varphi(d)=n / \varphi(n)(n \geq 1)$ fournit

$$
\begin{aligned}
\sum_{n>z} \frac{1}{n^{\beta} \varphi(n)} & =\sum_{n>z} \frac{1}{n^{1+\beta}} \sum_{d \mid n} \frac{\mu(d)^{2}}{\varphi(d)}=\sum_{d \geq 1} \frac{\mu(d)^{2}}{\varphi(d)} \sum_{\substack{n>z \\
n \equiv 0 \bmod d}} \frac{1}{n^{1+\beta}} \\
& \ll \frac{1}{\beta z^{\beta}} \sum_{d \geq 1} \frac{\mu(d)^{2}}{d \varphi(d)} \ll \frac{1}{\beta z^{\beta}} .
\end{aligned}
$$

3. Démonstration du Théorème 1. De manière à pouvoir évaluer des sommes sur les entiers friables translatés, nous sommes amenés à traiter séparément les contributions des entiers friables dans les progressions arithmétiques de petits modules - pour lesquels l'on dispose de résultats de type Bombieri-Vinogradov - et de grands modules.

Nous donnons ainsi les étapes de la démonstration sous forme d'énoncés distincts.

Lemme 11. Soit $f$ une fonction multiplicative telle que $\lambda:=f * \mu$ vérifie (6). Nous avons, uniformément pour tous $x, y$ satisfaisant $\left(\mathrm{H}_{\varepsilon}\right)$ et $\beta>0$,

$$
\sum_{d \leq z} \frac{\lambda(d)}{\varphi(d)} \Psi_{d}(x, y)=\Psi(x, y)\left\{C(f)+O\left(\frac{\log (u+1)}{\log y}+\frac{1}{\beta z^{\beta}}\right)\right\},
$$

où la constante implicite dépend au plus de B.

Démonstration. Intervertissant les sommations, nous obtenons, pour $z \geq 2$,

$$
\begin{aligned}
S & =\sum_{d \leq z} \frac{\lambda(d)}{\varphi(d)} \Psi_{d}(x, y)=\sum_{n \in S(x, y)} \sum_{\substack{d \leq z \\
(n, d)=1}} \frac{\lambda(d)}{\varphi(d)} \\
& =\sum_{n \in S(x, y)}\left\{\sum_{\substack{d \geq 1 \\
(n, d)=1}} \frac{\lambda(d)}{\varphi(d)}+O\left(\sum_{d>z} \frac{|\lambda(d)|}{\varphi(d)}\right)\right\},
\end{aligned}
$$

où les séries sont convergentes puisque $|\lambda(d)| / \varphi(d) \ll 1 / d^{1+\beta / 2}(d \geq 1)$ sous la condition (6).

Posons $G_{p}:=\sum_{\nu \geq 0} \lambda\left(p^{\nu}\right) / \varphi\left(p^{\nu}\right)(p \geq 2)$. La condition (6) implique encore

$$
\left|G_{p}-1\right|=\left|\sum_{\nu \geq 1} \frac{\lambda\left(p^{\nu}\right)}{\varphi\left(p^{\nu}\right)}\right| \leq \sum_{\nu \geq 1} \frac{B}{p^{\beta \nu} \varphi\left(p^{\nu}\right)} \leq \frac{4 B}{p^{1+\beta}} \quad(p \geq 2) .
$$

Cette relation implique en particulier que $G_{p} \neq 0$ pour $p \geq 4 B$, et ainsi que $G_{p}$ n'est négatif ou nul que pour au plus un nombre fini de valeurs de $p$. Il 
vient donc

$$
\left|\frac{1}{G_{p}}-1\right| \leq \frac{8 B}{p^{1+\beta}} \quad(p \geq 8 B)
$$

L'estimation (27) assure la convergence des produits $C_{1}:=\prod_{G_{p} \neq 0} G_{p}$ et

$$
\prod_{p \nmid n} G_{p}=\sum_{\substack{d \geq 1 \\(n, d)=1}} \frac{\lambda(d)}{\varphi(d)}
$$

Nous avons ainsi, par le Lemme 10.

$$
S=\sum_{n \in S(x, y)} \prod_{p \nmid n} G_{p}+O\left(\frac{\Psi(x, y)}{\beta z^{\beta}}\right) .
$$

Les seuls termes non nuls de la dernière somme friable sont ceux pour lesquels l'indice $n$ est divisible par $Q:=\prod_{G_{p}=0} p$, où le produit est fini, comme indiqué précédemment. Ainsi, nous avons

$$
S=C_{1} \sum_{n \in S(x / Q, y)} h(n)+O\left(\frac{\Psi(x, y)}{\beta z^{\beta}}\right), \quad \text { où } \quad h(n):=\prod_{\substack{p \mid n \\ G_{p} \neq 0}} \frac{1}{G_{p}} .
$$

La fonction $h$ vérifie les hypothèses (10) et (11). Les deux estimations étant semblables, bornons-nous à montrer que $h$ vérifie (11). Nous avons, pour $z \geq 2$, grâce à la relation (28),

$$
\begin{aligned}
\sum_{\substack{p \leq z \\
h(p)<0}}|h(p)| \log p+\left|\sum_{p \leq z} h(p) \log p-z\right| & \ll 1+\left|\sum_{p \leq z} \log p-z\right|+\sum_{p \leq z} \frac{1}{p^{1+\beta}} \\
& \ll \frac{z}{L_{\varepsilon}(z)}+\log _{2} z \ll \frac{z}{L_{\varepsilon}(z)},
\end{aligned}
$$

où les constantes implicites ne dépendent que de $B$.

Nous pouvons donc évaluer la somme friable de la fonction multiplicative $h$ par le Lemme 6. Nous avons alors, pour $x, y$ vérifiant $\left(\mathrm{H}_{\varepsilon}\right)$,

$$
\sum_{n \in S(x / Q, y)} h(n)=\Psi\left(\frac{x}{Q}, y\right)\left\{C(h)+O\left(\frac{\log (u+1)}{\log y}\right)\right\} .
$$

Par ailleurs, le Lemme 5 et l'estimation (7) nous assurent que si $(x, y)$ est dans le domaine $\left(\mathrm{H}_{\varepsilon}\right)$ et si $Q$ vérifie $\log (Q+1) \leq(\log y) / \log (u+1)$, nous avons

$$
\Psi\left(\frac{x}{Q}, y\right)=\frac{\Psi(x, y)}{Q}\left\{1+O\left(\frac{\log (Q+1) \log (u+1)}{\log y}+\frac{\log Q}{\log x}\right)\right\}
$$


et ainsi

$$
\sum_{n \in S(x / Q, y)} h(n)=\Psi(x, y)\left\{\frac{C(h)}{Q}+O\left(\frac{\log (Q+1) \log (u+1)}{Q \log y}\right)\right\} .
$$

L'égalité $\varphi\left(p^{\nu}\right)=p^{\nu}(1-1 / p)(p \geq 2, \nu \geq 1)$ permet d'établir la relation

$$
G_{p}=E_{f}(p)+\frac{E_{f}(p)-1}{p-1} \quad(p \geq 2),
$$

qui nous assure que l'on a $G_{p}=0$ si et seulement si $E_{f}(p)=1 / p$. De plus, la relation (6) implique que le produit $C(f)$ est convergent (voir par exemple le $\S$ I.3.8 des notes de [10]). Par conséquent,

$$
\frac{C_{1} C(h)}{Q}=\prod_{E_{f}(p) \neq 1 / p} G_{p}\left(1-\frac{1}{p}\right)\left(1+\frac{G_{p}^{-1}}{p-1}\right) \prod_{E_{f}(p)=1 / p} \frac{1}{p}=\prod_{p} E_{f}(p) .
$$

Nous avons finalement, pour $(x, y) \in\left(\mathrm{H}_{\varepsilon}\right)$,

$$
S=\Psi(x, y)\left\{C(f)+O\left(\frac{\log (u+1)}{\log y}+\frac{1}{\beta z^{\beta}}\right)\right\} \text {. }
$$

LEMME 12. Soit $\lambda$ une fonction arithmétique vérifiant la condition (6). Il existe une constante $c_{7}:=c_{7}(\beta, B)>0$ telle que, pour

$$
(x, y) \in\left(\mathrm{H}_{\varepsilon}\right), \quad c_{7}(\log x)^{3 / \beta} \leq z \leq x,
$$

nous ayons uniformément

$$
\sum_{z<d \leq x}|\lambda(d)| \Psi(x, y ; 1, d) \ll \frac{\Psi(x, y)}{\log x} .
$$

Démonstration. Posons $Z:=\exp \left\{y^{\beta / 8}\right\}$. En appliquant la majoration (24), l'estimation $1 / \varrho(u) \ll \exp \left\{u \log u+O\left(u \log _{2} u\right)\right\}(u \geq 3)$ et la méthode de Rankin, nous avons, pour $(x, y) \in\left(\mathrm{H}_{\varepsilon}\right)$,

$$
\begin{aligned}
\sum_{z<d \leq Z}|\lambda(d)| \Psi(x, y ; 1, d) & \leq \Psi(x, y) \sum_{z<d \leq Z} \frac{B}{d^{1+\beta}} d^{2\left(\log _{2} d\right) / \log y}(\log d)^{2} \\
& \ll \frac{\Psi(x, y)}{z^{\beta / 3}} \sum_{1 \leq d \leq Z} \frac{1}{d^{1+\beta / 3}} \ll \frac{\Psi(x, y)}{\beta z^{\beta / 3}},
\end{aligned}
$$

compte tenu de l'hypothèse $(6)$. La majoration triviale $\Psi(x, y ; 1, d) \ll x / d$ fournit par ailleurs

$$
\sum_{Z<d \leq x}|\lambda(d)| \Psi(x, y ; 1, d) \ll x \sum_{Z<d} \frac{|\lambda(d)|}{d} \ll \frac{x}{\beta Z^{\beta / 3}} \ll \frac{\Psi(x, y)}{\log x},
$$

où la dernière inégalité est clairement vérifiée dans le domaine $\left(\mathrm{H}_{\varepsilon}\right)$. 
Fin de la démonstration du Théorème 1. Utilisons la relation de convolution $f=\lambda * 1$. Il vient, pour tout $z \geq 2$,

où

$$
\Theta_{f}(x, y)=\sum_{d \leq x} \lambda(d) \Psi(x, y ; 1, d)=S_{1}+S_{2}+S_{3},
$$

$$
\begin{aligned}
S_{1} & :=\sum_{d \leq z} \frac{\lambda(d)}{\varphi(d)} \Psi_{d}(x, y), \\
S_{2} & :=\sum_{d \leq z} \lambda(d) E(x, y ; 1, d), \\
S_{3} & :=\sum_{z<d \leq x} \lambda(d) \Psi(x, y ; 1, d) .
\end{aligned}
$$

Le Lemme 11 fournit, pour le choix de $z$ effectué au Lemme 8 ,

$$
S_{1}=\Psi(x, y)\left\{C(f)+O\left(\frac{\log (u+1)}{\log y}\right)\right\} \quad\left((x, y) \in\left(\mathrm{H}_{\varepsilon}\right)\right) .
$$

Le Lemme 8 conduit à la majoration

$$
\left|S_{2}\right| \ll \sum_{d \leq z}|E(x, y ; 1, d)| \ll \frac{\Psi(x, y)}{\log x} \quad\left((x, y) \in\left(\mathrm{H}_{\varepsilon}\right)\right) .
$$

Enfin, nous déduisons du Lemme 12 que nous avons une majoration du même ordre de grandeur pour $S_{3}$, dans le domaine $\left(\mathrm{H}_{\varepsilon}\right)$. En effet, la condition (29) du Lemme 12 est, compte tenu du choix du paramètre $z$, remplie dans ce domaine.

\section{Preuves des corollaires}

4.1. Démonstration du Corollaire 2. Nous avons, pour $x, y$ satisfaisant $\left(\mathrm{H}_{\varepsilon}\right)$,

$$
\Theta_{F}(x, y)=\sum_{\substack{1<n \leq x \\ n \in S(x, y)}}(n-1) f(n-1)=(x-1) \Theta_{f}(x, y)-\int_{1}^{x} \Theta_{f}(t, y) d t
$$

Posons $E_{u, y}:=\{\log (u+1)\} / \log y$. Le Théorème 1 impliquant trivialement

$$
\Theta_{f}(t, y)=\Psi(t, y)\left\{C(f)+O\left(E_{u, y}+\frac{1}{\log 2 t}\right)\right\} \quad(1 \leq t \leq x),
$$

il vient, en vertu du Lemme 7 ,

$$
\begin{aligned}
\int_{1}^{x} \Theta_{f}(t, y) d t & =\int_{1}^{x} \Psi(t, y) d t\left\{C(f)+O\left(E_{u, y}\right)\right\}+O\left(\int_{1}^{y} \frac{t d t}{\log 2 t}\right) \\
& =x \Psi(x, y)\left\{\frac{1}{2} C(f)+O\left(E_{u, y}\right)\right\} .
\end{aligned}
$$


En effet, comme $x \geq y$, le terme $\int_{1}^{y} t d t / \log 2 t \ll y^{2} / \log y \ll x \Psi(x, y) / \log y$ est clairement englobé par le dernier terme d'erreur de (30).

Nous pouvons ainsi déduire des estimations précédentes que

$$
\begin{aligned}
\Theta_{F}(x, y) & =(x-1) \Psi(x, y)\left\{C(f)+O\left(E_{u, y}\right)\right\}-x \Psi(x, y)\left\{\frac{1}{2} C(f)+O\left(E_{u, y}\right)\right\} \\
& =x \Psi(x, y)\left\{\frac{1}{2} C(f)+O\left(E_{u, y}\right)\right\} .
\end{aligned}
$$

4.2. Démonstration du Corollaire 3, L'identité de convolution $\varphi(n) / n=\sum_{d \mid n} \mu(d) / d(n \geq 1)$ montre que la fonction $d \mapsto \mu(d) / d$ vérifie trivialement la condition (6) du Théorème 1. De plus, nous avons

$$
C(\varphi / j)=\prod_{p}\left(1-\frac{1}{p^{2}}\right)=\frac{6}{\pi^{2}} .
$$

D'après le Corollaire 2, cela fournit l'estimation annoncée pour $\Theta_{\varphi}(x, y)$.

Il en va de même pour $\Theta_{\sigma}(x, y)$, grâce aux relations $\sigma(n) / n=\sum_{d \mid n} 1 / d$ $(n \geq 1)$ et

$$
C(\sigma / j)=\prod_{p}\left(1-\frac{1}{p^{2}}\right)^{-1}=\frac{\pi^{2}}{6} .
$$

Par ailleurs, nous avons $\varphi_{1} / j=\lambda_{1} * \mathbf{1}$, avec

$$
\lambda_{1}(2)=\frac{1}{2}, \quad \lambda_{1}(p)=-\frac{1}{p} \quad(p \geq 3), \quad \lambda_{1}\left(p^{\nu}\right)=0 \quad(p \geq 2, \nu \geq 2) .
$$

Le Corollaire 2 permet donc encore de conclure dans le cas de $\varphi_{1}$.

Enfin, il est immédiat que l'on ait $C\left(f_{1}\right)=0$ et que $f_{1}$ vérifie la condition (6) : cela fournit bien l'estimation attendue, grâce au Théorème 1.

Remerciements. L'auteur tient ici à exprimer sa gratitude envers Gérald Tenenbaum pour son aide précieuse tout au long de l'élaboration de cet article.

\section{Bibliographie}

[1] R. de la Bretèche et G. Tenenbaum, Propriétés statistiques des entiers friables, Ramanujan J. 9 (2005), 139-202.

[2] É. Fouvry, Sur le problème des diviseurs de Titchmarsh, J. Reine Angew. Math. 357 (1985), 51-76.

[3] É. Fouvry et G. Tenenbaum, Diviseurs de Titchmarsh des entiers sans grand facteur premier, dans : Analytic Number Theory (Tokyo, 1988), Lecture Notes in Math. 1434, Springer, Berlin, 1990, 86-102.

[4] A. Granville, Integers, without large prime factors, in arithmetic progressions, I, Acta Math. 170 (1993), 255-273.

[5] A. Hildebrand, On the number of positive integers $\leq x$ and free of large prime factors $>$ y, J. Number Theory 22 (1986), 289-307.

[6] A. Hildebrand and G. Tenenbaum, On a class of differential-difference equations arising in number theory, J. Anal. Math. 61 (1993), 145-179. 
[7] S. Loiperdinger and I. Shparlinski, On the distribution of the Euler function of shifted smooth numbers, Colloq. Math. 120 (2010), 139-148.

[8] H. Smida, Sur les puissances de convolution de la fonction de Dickman, Acta Arith. 59 (1991), 123-143.

[9] - Valeur moyenne des fonctions de Piltz sur les entiers sans grand facteur premier, ibid. 63 (1993), 21-50.

[10] G. Tenenbaum, Introduction à la théorie analytique et probabiliste des nombres, $3^{\text {ème }}$ éd., Berlin, Paris, 2008.

[11] G. Tenenbaum et J. Wu, Moyennes de certaines fonctions multiplicatives sur les entiers friables, J. Reine Angew. Math. 564 (2003), 119-166.

Joseph Basquin

Institut Élie Cartan

Université Henri Poincaré-Nancy 1

BP 239

54506 Vandœuvre Cedex, France

E-mail: joseph.basquin@iecn.u-nancy.fr

Reçu le 23.11.2009

et révisé le 18.2.2010 